\title{
Practical insights for drug development using the animal rule
}

\begin{abstract}
The Food and Drug Administration's (FDAs) Medical Countermeasures Initiative was launched in 2010. The initiative is intended to coordinate health care responses to potential chemical, biological, radiological, nuclear, and emerging infectious disease threats by ensuring that the medical countermeasures (eg, drugs, vaccines, and diagnostic tests) that are developed are safe, effective, and secure. The incentives for developing these medical counter-measures include the potential to secure a Priority Review Voucher under the 21st Century Cures Act and the potential for an extended 5 years of exclusivity for the chemical entity. These incentives have increased interest in medical countermeasures and in the use of the Animal Rule to demonstrate substantial evidence of efficacy. This Mini Review offers practical insights into the purpose, challenges, and benefits of using of the Animal Rule as the regulatory route for new drug approval. The information presented is derived from the FDAs Guidance document.
\end{abstract}

Keywords: animal rule, medical countermeasures, biothreats, $21^{\text {st }}$ century cures act, priority review voucher
Volume 3 Issue I - 2017

\author{
William R Kelce, Lavonne M Patton \\ Cardinal Health Regulatory Sciences, USA
}

Correspondence: William R Kelce, Cardinal Health Regulatory Sciences, 7400 West I I0th Street, Ste 300, Overland Park, KS 662 I0, Tel 91366/2040, Fax 91345|3846,

Email william.kelce@cardinalhealth.com

Received: February 01, 2016 | Published: February 09, 2017

\section{Introduction}

In response to the terror attacks in 2001 involving anthrax spores sent through the mail, the United States government established a regulatory process whereby potential countermeasure treatments intended to protect the safety of the public could be tested for safety and efficacy using laboratory animals. This conditional approval process, called the Animal Rule, was critical as it is not ethical to expose humans to chemical, biological, radiological, or nuclear substances in order to establish efficacy of potential medical countermeasures. Sponsors cannot use this regulatory pathway when human safety and efficacy data can be obtained for a relevant human indication using a traditional regulatory pathway. The regulatory approval requires post marketing studies, restrictions to ensure safe use, and labeling to indicate that approval was based on efficacy studies in animals. The use of the Animal Rule as a regulatory pathway to conditional approval, is intended to reduce or prevent serious and life-threatening conditions caused by chemical, biological, radiological, or nuclear substances regardless of whether exposure was deliberate (eg, terrorist use of nerve agent or Bacillus anthracis) or accidental (eg, emerging infectious pathogens, snake venom, industrial chemicals). Food and Drug Administration regulations for the approval of new drugs under the Animal Rule are codified in 21 CFR 314.600 through 314.650 for drugs and 21 CFR 601.90 through 601.95 for biological products.

\section{Practical insights to using the animal rule}

1. The FDA's Animal Rule is used for the development of drug and biological products to treat serious or life-threatening conditions when human efficacy studies are not ethical or feasible per other FDA regulations.

2. The Animal Rule allows adequate and well-controlled animal studies to be used as evidence of effectiveness.

3. The mechanisms by which the toxic agent and the protective drug act should be reasonably well understood.
4. The animal model should be predictive of clinical benefit in humans.

5. The animal data should allow selection of an effective dose in humans.

6. Drugs approved under the Animal Rule must also be evaluated for safety using traditional approaches (eg, animal studies and healthy human volunteer studies).

7. Approval of a drug or biological product under the Animal Rule imposes requirements to submit plans for additional postmarketing safety and efficacy studies, restrictions to ensure safe use by health care practitioners, and prescription labeling to indicate that regulatory approval was based on efficacy studies in animals.

8. The Food and Drug Administration will take into account all existing data with the drug or biological product to determine whether the animal data provide substantial evidence of effectiveness.

9. The Animal Rule does not apply if approval is possible by other routes such as a related indication.

10. The protocols to demonstrate efficacy in animals are eligible for evaluation under a special protocol assessment (SPA).

11. The Animal Rule development program may be eligible for expedited development under Fast Track and Priority Review designation programs.

12. The development of medical countermeasures may qualify for a Priority Review Voucher under the 21st Century Cures Act and for an extended 5 years of exclusivity for the chemical entity.

13. Early discussions with FDA regarding the use of the Animal Rule for regulatory approval are critical for success. 
The FDAs Medical Countermeasures Initiative, under the Office of Counterterrorism and Emergency Coordination, has no regulatory authority and can work openly with sponsors to provide feedback on pre-investigational new drug (pre-IND) and IND applications to:

A. Help define the field use of the countermeasure being developed.

B. Help refine questions to CDER/CBER.

C. Provide feedback on the proposed animal models.

D. Discussion of the target product profile with FDA early in development is strongly recommended to keep the focus on key issues throughout the approval process.

\section{Challenges to approval using the animal rule}

Development under the Animal Rule must meet traditional approval requirements for safety, plus the Sponsor must design a nonclinical program to provide substantial evidence of effectiveness. The Food and Drug Administration can use animal data as substantial evidence of effectiveness only when all 4 of the following criteria are met:

a. The pathophysiological mechanism of the toxic agent, and the protective mechanism(s) by which the drug acts, are reasonably well understood;

b. Efficacy is demonstrated in more than 1 animal species expected to be predictive for humans or in 1 animal species known to be predictive;

c. The animal study endpoint is clearly related to the desired benefit in humans (eg, enhanced survival or prevention of major morbidity); and

d. Animal data (eg, pharmacokinetics, pharmacodynamics) allow selection of an effective dose in humans.

The rationale for the use of the Animal Rule must be supported by proof-of-concept animal studies designed to ensure animal welfare, demonstrate relevance of the animal model (species susceptibility to the toxic agent and toxic response similar to humans), define the endpoint used to assess efficacy and predict human clinical benefit (eg, relief or prevention of adverse effects), ensure data quality and integrity in a contained environment, and be conducted under Good Laboratory Practice (GLP) guidelines. When efficacy is demonstrated in a single study, conducted in a sufficiently well-characterized animal model, it may be necessary to repeat the study, preferably in another laboratory for confirmation. The investigational drug/biologic used in the adequate and well-controlled animal efficacy studies and in the animal pharmacokinetics and/or pharmacodynamics studies used to select a dose and dosing regimen in humans, ideally should be manufactured under current GMP regulations and be as close as practicable to the to-be-marketed drug/biologic.

\section{Benefit to the sponsor}

Products developed under the Animal Rule have the potential to be available to the public earlier and use fewer resources, compared to approval through the traditional regulatory pathway. Additional benefits associated with expedited programs include accelerated reviews, more frequent guidance and communication with FDA, potential for earlier marketing of the drug/biologic, eligibility for a Priority Review Voucher, potential for an additional 5 years of exclusivity for the chemical entity.

\section{Products approved using the animal rule}

Sponsors are encouraged to review the summary basis of approval information, available at FDA's website, for products that have been previously approved under the Animal Rule for additional valuable insight. Drug products approved to date under the animal rule are listed in the table below together with the indication and the animal species used to establish substantial evidence of effectiveness (Table $1)$.

Table I

\begin{tabular}{|c|c|c|}
\hline \multicolumn{3}{|c|}{ Drug products and development programs approved under the animal rule ${ }^{a}$} \\
\hline Drug product (Year) & Brief indication & $\begin{array}{l}\text { Animal species } \\
\text { used to establish } \\
\text { efficacy }\end{array}$ \\
\hline Pyridostigmine bromide (2003) & $\begin{array}{l}\text { For use as a pretreatment for exposure to the chemical nerve agent } \\
\text { Soman }\end{array}$ & $\begin{array}{l}\text { Nonhuman primates, } \\
\text { guinea pigs, and } \\
\text { rodents }\end{array}$ \\
\hline $\begin{array}{l}\text { Cyanokit (hydroxocobalamin) } \\
\text { (2006) }\end{array}$ & For treatment of known or suspected cyanide poisoning & Dogs \\
\hline Levaquin (levofloxacin) (2012) & For prophylaxis and treatment of plague & Nonhuman primates \\
\hline Raxibacumab (20I2) & $\begin{array}{l}\text { For treatment of inhalational anthrax in combination with antibacterial } \\
\text { drugs }\end{array}$ & $\begin{array}{l}\text { Nonhuman primates } \\
\text { and rabbits }\end{array}$ \\
\hline $\begin{array}{l}\text { Botulism Antitoxin Heptavalent (A, } \\
\text { B, C, D, E, F, G)-(Equine) (2013) }\end{array}$ & $\begin{array}{l}\text { For treatment of patients showing signs of botulism following documented } \\
\text { or suspected exposure to botulinum neurotoxin }\end{array}$ & $\begin{array}{l}\text { Nonhuman primates } \\
\text { and guinea pigs }\end{array}$ \\
\hline Ciprofloxacin (2015) & For the prophylaxis and treatment of plague & Nonhuman primates \\
\hline Moxifloxacin (Avelox) (2015) & For the prophylaxis and treatment of plague & Nonhuman primates \\
\hline
\end{tabular}


Table continued..

Drug products and development programs approved under the animal rule ${ }^{a}$

\begin{tabular}{|c|c|c|}
\hline Drug product (Year) & Brief indication & $\begin{array}{l}\text { Animal species } \\
\text { used to establish } \\
\text { efficacy }\end{array}$ \\
\hline $\begin{array}{l}\text { Anthrax Immune Globulin } \\
\text { Intravenous (Anthrasil) (20I5) }\end{array}$ & $\begin{array}{l}\text { For the treatment of inhalational anthrax in adult and pediatric patients in } \\
\text { combination with appropriate antibacterial drugs }\end{array}$ & $\begin{array}{l}\text { Nonhuman primates } \\
\text { and rabbits }\end{array}$ \\
\hline Filgrastim (Neupogen) (20I5) & $\begin{array}{l}\text { For increased survival in patients acutely exposed to myelosuppressive } \\
\text { doses of radiation (Hematopoietic Syndrome of Acute Radiation } \\
\text { Syndrome) }\end{array}$ & Nonhuman primates \\
\hline Pegfilgrastim (Neulasta) (20I5) & For adult and pediatric patients at risk of developing myelosuppression & Nonhuman primates \\
\hline $\begin{array}{l}\text { Anthrax Vaccine Absorbed } \\
\text { (BioThrax) (20I5) }\end{array}$ & For use after known or suspected anthrax exposure & $\begin{array}{l}\text { Nonhuman primates } \\
\text { and rabbits }\end{array}$ \\
\hline Obiltoxaximab (Anthim) $)^{\mathrm{b}}(2016)$ & For treatment of adult and pediatric patients exposed to inhaled anthrax ${ }^{b}$ & $\begin{array}{l}\text { Nonhuman primates } \\
\text { and rabbits }\end{array}$ \\
\hline
\end{tabular}

$\mathrm{a}=$ Mac Gill T (2016) The FDA Animal Rule.

b=King S (2016) Regulatory Perspective on the Use of Animal Models of Disease in Drug Development.

\section{Acknowledgements}

None.

\section{Conflict of interest}

The author declares no conflict of interest.

\section{References}

1. US Food and Drug Administration. Product Development Under the Animal Rule Guidance for Industry. USA: Silver Spring; 2015. p. 1-54. 